\title{
PERFIL SOCIODEMOGRÁFICO E TRABALHISTA DOS TRABALHADORES RURAIS VÍTIMAS DE ACIDENTE NO SEMIÁRIDO CEARENSE
}

Francisco Rosemiro Guimarães Ximenes Netoํ․, Dyêgo de Oliveira Aurélio¹, Francisco Diogenes dos Santos ${ }^{1}$, Verena Emmanuelle Soares Ferreira², Rafaela Almeida Ribeiro Pereira ${ }^{2}$, Maria Socorro Carneiro Linhares ${ }^{3}$

Objetivo: descrever o perfilsociodemográfico e trabalhista dos trabalhadores rurais vítimas de acidentes de trabalho grave. Metodologia: estudo epidemiológico, descritivo e retrospectivo, realizado no Centro Regional de Referência em Saúde do Trabalhador (CEREST) de Sobral - Ceará, Brasil, com 509 casos de acidentes de trabalho com trabalhadores rurais, notificados pelo Sistema Nacional de Agravos de Notificação (SINAN), entre 1ㅇ de janeiro de 2009 a 31 de dezembro de 2013. Resultados: 98\% (500) são do sexo masculino; $79,6 \%$ (405) estão na faixa etária entre 20 e 59 anos; 92\% (472) são pardos; 32,2\% (164) tem ensino fundamental I incompleto; 48,9\% (249) estão em situação de trabalho precarizado. Conclusão: o panorama dos acidentes com trabalhadores rurais evidencia a necessidade de uma melhor atenção por parte do Setor Saúde e das representações dessa classe trabalhadora.

Descritores: Epidemiologia descritiva, Saúde do Trabalhador, Acidentes de Trabalho, Agricultor.

\section{SOCIODEMOGRAPHIC AND LABOR PROFILE OF RURAL WORKERS VICTIMS OF ACCIDENTS IN CEARÁ}

Objective: to describe the sociodemographic and labor profile of rural workers victims of serious accidents. Methodology: epidemiological, descriptive and retrospective study carried out in the Occupational Health Reference Regional Centre (CEREST), in Sobral - Ceará, Brazil, with 509 cases of occupational accidents with rural workers, reported by the National Notifiable Diseases System (SINAN) between 1 January 2009 to 31 December 2013. Results: 98\% (500) were male; 79.6\% (405) are aged between 20 and 59 years; 92\% (472) are brown; 32.2\% (164) have incomplete elementary school; 48.9\% (249) are in precarious work situation. Conclusion: the panorama of accidents with rural workers highlights the need for better attention from the health sector, and the representations of this working class.

Descriptors: Descriptive epidemiology, Worker's health, Work accidents, Farmer.

\section{PERFILSOCIODEMOGRÁFICOY LABORALDE LOSTRABAJADORES RURALES VÍCTIMAS DEACCIDENTES EN ELSEMIÁRIDO CEARENSE}

Objetivo: describir el perfil sociodemográfico y laboral de los trabajadores rurales víctimas de accidentes de trabajo graves. Metodologia: estudio epidemiológico, descriptivo y retrospectivo, realizado en el Centro Regional de Referencia en Salud del Trabajador (CEREST) de Sobral - Ceará, Brasil, con 509 casos de accidentes de trabajo con trabajadores rurales, notificados por el Sistema Nacional de Agravamientos de Notificación (SINAN), entre 1ㅇ de enero de 2009 y 31 de diciembre de 2013. Resultados: 98\% (500) son de sexo masculino; 79,6\% (405) están en la franja de edad de 20 a 59 años; 92\% (472) son pardos; 32,2\% (164) tienen enseñanza fundamental I incompleta; 48,9\% (249) están en situación de trabajo precario. Conclusión: el panorama de los accidentes con trabajadores rurales evidencia la necesidad de una mayor atención por parte del Sector de Salud y de las representaciones de esta clase trabajadora. Descriptores: Epidemiología descriptiva, Salud del Trabajador, Accidentes de Trabajo, Agricultor.

IEnfermeiro. Universidade Estadual Vale do Acaraú (UVA), Sobral - Ceará, Brasil. rosemironeto@gmail.com

${ }^{2}$ Enfermeiro. Centro Regional de Referência em Saúde do Trabalhador (CEREST) /Secretaria da Saúde, Sobral - Ceará, Brasil ${ }^{3}$ Enfermeiro. Universidade Estadual Vale do Acaraú (UVA), Sobral - Ceará, Brasil 


\section{INTRODUÇÃO}

No Brasil, ocorrem transformações em sua base produtiva no último século, principalmente, nos componentes políticos, sociais, econômicos e de proteção legal e trabalhista, por conta de mudanças na economia local e influências da tendência do mercado mundial. Essas transformações demandam uma análise do trabalho, transcendendo a clássica divisão econômica de setores primário, secundário e terciário(1); situação esta intensificada na década de 1960, o que repercutiu negativamente nas condições de vida, trabalho e na saúde do trabalhador rural(2).

Contudo, os processos produtivos predominantes no meio rural vivenciam uma situação diversa de produção e racionalidades, que vão desde o modo histórico artesanal do uso de ferramentas como enxadas, machado, foice, facas e facões na agricultura familiar (dos minifúndios), até a densa mecanização em diversas regiões e setores agrícolas. Bancados pelas elites agrárias (dos latifúndios), expõem trabalhadores e trabalhadoras rurais a diversos riscos, agravos e acidentes durante o labor, sejam com ferramentas manuais, ou intoxicação e envenenamento por agrotóxico.

A análise de pesquisa ${ }^{(3)}$ aponta que 50\% a 70\% desses trabalhadores estão expostos a fatores de risco (ergonômicos, biológicos, físicos, químicos e psicossociais); mais de $60 \%$ sofrem com as cargas excessivas de trabalho: e $80 \%$ têm exposição a ruídos, vibrações, radiações iônicas, eletromagnéticas e a microclimas insalubres no local de trabalho; exposição a outros agentes causadores de doenças, como as máquinas e equipamentos agrícolas, ferramentas manuais, agrotóxicos, dentre outros. Na maioria dos procedimentos, os trabalhadores rurais não utilizam equipamentos de proteção individual (EPI), o que predispõe a mais riscos.

Ainda na zona rural, a maioria dos trabalhadores rurais trabalham por conta própria e, ao sofrerem acidentes, não procuram os serviços de saúde para buscarem uma atenção especializada e registrarem o acidente de trabalho sofrido, o que contribui, de certa forma, a um limitado número de notificações. Estudo acerca das evidências sobre as iniquidades sanitárias aponta que mais de $50 \%$ da população rural do mundo apresentam dificuldades de acesso aos cuidados de saúde( ${ }^{(4)}$, o que pode contribuir para a nao notificação dos acidentes.

Nesse sentido, como estratégia de melhorar a atenção e a vigilância à saúde dos trabalhadores no Brasil, nos diversos setores da economia e ocupações, o Ministério da Saúde institucionaliza a "Política Nacional de Saúde do Trabalhador e da Trabalhadora" no Sistema Único de Saúde (SUS), baseada em unidades sentinelas, como os Centros de Referência de Saúde do Trabalhador (CEREST) ${ }^{(5)}$. Destaca-se ainda a criação da "Política Nacional de Saúde Integral das Populações do Campo, da Floresta e Águas", com o intento de melhorar as condições de saúde e a qualidade de vida dessas populações, considerando a saúde na sua complexidade e respeitando as especificidades do contexto em que esses grupos estão inseridos ${ }^{(6)}$

Considerando a situação de risco e vulnerabilidade que vivenciam os trabalhadores rurais durante suas atividades laborais, bem como a dificuldade de acesso destes aos serviços de saúde, este estudo tem como objetivo descrever o perfil sociodemográfico e trabalhista dos trabalhadores rurais vitimas de acidentes de trabalho grave.

\section{METODOLOGIA}

Estudo epidemiológico, descritivo e retrospectivo, realizado no CEREST de Sobral - Ceará, entre maio de 2014 e janeiro de 2015, com 509 casos de acidentes de trabalho grave com trabalhadores rurais, notificados no Sistema Nacional de Agravos de Notificação (SINAN), entre 1o de janeiro de 2009 e 31 de dezembro de 2013, nos 47 municípios que compõem o referido CEREST. Os casos estão assim distribuídos por ano de ocorrência: 2009 - 43 (8,4\%); 2010 - 80 (15,8\%); 2011 - 108 (21,2\%); 2012 - 129 (25,3\%); e 2013 - $149(29,3 \%)^{(7)}$.

Os dados secundários foram extraídos do banco de dados do SINAN dos municípios da área de abrangência do CEREST, depois da autorização e assinatura do Termo de Fiel Depositário. Estes foram organizados por meio de planilhas eletrônicas, geradas pelo sistema do programa TabWin32 versão 3.6b e exportadas para o programa Excel 2007, e sistematizados as partir das categorias de variáveis, selecionadas na "Ficha de Acidente de Trabalho Grave"(8) do Ministério da Saúde, que são: sexo; idade; raça; escolaridade e situação no mercado de trabalho. Os dados estão apresentados em forma tabular, com cálculos dos números absolutos e frequências percentuais simples dos eventos estudados, com a estratificação por sexo e idade.

Estudo aprovado pelo Comitê de Ética em Pesquisa, parecer № 1.344.0660, conforme Resolução № 466/2012 do Conselho Nacional de Saúde. Esta pesquisa é um recorte da pesquisa intitulada "Doença, labor e trabalho no Semiárido Cearense: avalição do perfil dos acidentes e da mortalidade por causas relacionadas ao trabalho na Zona Norte do Ceará, 2009 a 2013".

\section{RESULTADOS E DISCUSSÃO}

$\mathrm{Na}$ Tabela 1, estão descritas as características sociodemográficas dos trabalhadores rurais do estudo. 
Tabela 1 - Descrição dos dados sociodemográficos dos acidentes de trabalho com trabalhadores rurais. CEREST Regional de Sobral - Ceará, Brasil, 2009 a 2013; número total com estratificação por gênero e idade.

\begin{tabular}{|c|c|c|c|c|c|c|c|c|c|c|c|c|}
\hline \multirow{2}{*}{\multicolumn{3}{|c|}{ Categorias }} & \multicolumn{4}{|c|}{ Gênero } & \multicolumn{6}{|c|}{ Faixa Estária (anos) } \\
\hline & & & \multicolumn{2}{|c|}{$?$} & \multicolumn{2}{|l|}{$?$} & \multicolumn{2}{|c|}{10 a 19} & \multicolumn{2}{|c|}{20 a 59} & \multicolumn{2}{|c|}{60 e mais } \\
\hline Sexo & $\mathbf{N}$ & $\%$ & $\mathbf{N}$ & $\%$ & N & $\%$ & $\mathbf{N}$ & $\%$ & $\mathbf{N}$ & $\%$ & $\mathbf{N}$ & $\%$ \\
\hline Masculino & 500 & 98,0 & & & & & 47 & 92,1 & 402 & 99,2 & 50 & 96,1 \\
\hline Feminino & 9 & 2,0 & & & & & 4 & 7,9 & 3 & 0,8 & 2 & 3,9 \\
\hline Total & 509 & 100,0 & & & & & 51 & 100,0 & 405 & 100,0 & 52 & 100,0 \\
\hline \multicolumn{13}{|l|}{ Faixa Estária (anos) } \\
\hline Menos de 10 & 1 & 0,2 & 1 & 0,2 & 0 & 0,0 & & & & & & \\
\hline 10 a 14 & 5 & 1,0 & 4 & 0,8 & 1 & 11,0 & & & & & & \\
\hline 15 a 19 & 46 & 9,0 & 43 & 8,6 & 3 & 33,5 & & & & & & \\
\hline 20 a 34 & 155 & 30,6 & 155 & 31,0 & 1 & 11,0 & & & & & & \\
\hline 35 a 49 & 169 & 33,0 & 168 & 33,6 & 1 & 11,0 & & & & & & \\
\hline 50 a 64 & 106 & 21,0 & 103 & 20,6 & 3 & 33,5 & & & & & & \\
\hline 60 a 79 & 23 & 4,6 & 23 & 4,6 & 0 & 0,0 & & & & & & \\
\hline 80 e mais & 3 & 0,6 & 3 & 0,6 & 0 & 0,0 & & & & & & \\
\hline Total & 509 & 100,0 & 500 & 100,0 & 9 & 100,0 & & & & & & \\
\hline \multicolumn{13}{|l|}{ Raça/Cor } \\
\hline Parda & 472 & 92,7 & 464 & 92,8 & 8 & 88,8 & 48 & 94,0 & 375 & 92,6 & 48 & 92,4 \\
\hline Branca & 21 & 4,1 & 21 & 4,2 & 0 & 0,0 & 2 & 4,0 & 16 & 4,0 & 3 & 5,7 \\
\hline Preta & 10 & 2,0 & 10 & 2,0 & 0 & 0,0 & 0 & 0,0 & 10 & 2,4 & 0 & 0,0 \\
\hline Indigena & 3 & 0,6 & 2 & 0,4 & 1 & 11,1 & 1 & 2,0 & 2 & 0,5 & 0 & 0,0 \\
\hline Ignorado & 3 & 0,6 & 3 & 0,6 & 0 & 0,0 & 0 & 0,0 & 2 & 0,5 & 1 & 1,9 \\
\hline Total & 509 & 100,0 & 500 & 100,0 & 9 & 100,0 & 51 & 100,0 & 405 & 100,0 & 52 & 100,0 \\
\hline \multicolumn{13}{|l|}{ Escolaridade } \\
\hline Analfabeto & 35 & 6,9 & 35 & 7,0 & 0 & 0,0 & 1 & 2,0 & 28 & 7,0 & 6 & 11,5 \\
\hline Ensino Fundamental - I Incompleto & 164 & 32,2 & 162 & 32,4 & 2 & 22,2 & 6 & 11,8 & 132 & 32,6 & 25 & 48,2 \\
\hline Ensino Fundamental - I Completo & 25 & 4,9 & 25 & 5,0 & 0 & 0,0 & 1 & 2,0 & 22 & 5,4 & 2 & 3,8 \\
\hline Ensino Fundamental - II Incompleto & 63 & 12,4 & 62 & 12,4 & 1 & 11,1 & 12 & 23,5 & 47 & 11,6 & 4 & 7,7 \\
\hline Ensino Fundamental - II Completo & 12 & 2,4 & 12 & 2,4 & 0 & 0,0 & 0 & 0,0 & 11 & 2,7 & 1 & 1,9 \\
\hline Ensino Médio Incompleto & 11 & 2,2 & 11 & 2,2 & 0 & 0,0 & 5 & 9,8 & 6 & 1,5 & 0 & 0,0 \\
\hline Ensino Médio Completo & 17 & 3,3 & 15 & 3,0 & 2 & 22,2 & 3 & 5,9 & 12 & 3,0 & 2 & 3,8 \\
\hline Ensino Universitário Incompleto & 1 & 0,2 & 1 & 0,2 & 0 & 0,0 & 0 & 0,0 & 1 & 0,2 & 0 & 0,0 \\
\hline Ensino Universitário Completo & 2 & 0,4 & 2 & 0,4 & 0 & 0,0 & 0 & 0,0 & 2 & 0,5 & 0 & 0,0 \\
\hline Ignorado/Branco & 179 & 35,1 & 175 & 35,0 & 4 & 44,5 & 23 & 45,0 & 144 & 35,5 & 12 & 23,1 \\
\hline Total & 509 & 100,0 & 500 & 100,0 & 9 & 100,0 & 51 & 100,0 & 405 & 100,0 & 52 & 100,0 \\
\hline
\end{tabular}

Fonte: Sobral. Secretaria da Saúde. Centro de Referência em Saúde do Trabalhador (CEREST). Sistema Nacional de Agravos de Notificação (SINAN). 2014.7

A Tabela 1 mostra a predominância de homens, 500 (98\%), de diversas idades, que vão desde a infância à velhice, com uma prevalência da faixa etária de 20 a 59 anos (402 - 99,2\%). No trabalho rural, ainda, o homem é o donatário das mais diversas atividades, principalmente, as de maiores riscos, que demandam, tanto na agricultura, como na pecuária, grandes esforços e força física, ou seja, o exercício físico da atividade braçal, a exemplo do uso de ferramentas. Essa situação não minimiza a importância das mulheres no trabalho rural, apesar da invisibilidade destas, que labutam desde a madrugada até após o sol se por, diuturnamente, provendo a todos com alimentação e outros cuidados no lar, e ainda manufaturando diversos produtos da lavoura e do rebanho, para serem vendidos nos conglomerados urbanos.

A posição de subordinação da mulher na zona rural se assenta na divisão sexual do trabalho que se caracteriza na economia rural, assim como nas relações familiares de caráter patriarcal. Há dificuldade das próprias mulheres reconhecerem-se como trabalhadoras e produtoras, associadas, quase sempre, à situação de pobreza e exclusão em que vivem ${ }^{(9)}$.

Quanto à idade dos trabalhadores rurais, varia de nove a mais de 80 anos, com predominância de adultos jovens, entre a faixa etária de 20 a 49 anos, 63,6\% (325), fase essa em que os trabalhadores rurais apresentam maior energia, força e vitalidade para desempenhar suas atividades diárias, sejam na agricultura ou pecuária. A distribuição por faixa etária mostrou, ainda, que $10,2 \%$ (52) são crianças (1 $0,2 \%$ ) e adolescentes ( $51-10 \%$ ), e que $5,2 \%$ são idosos. As mulheres, apesar de um número limitado de casos, estão distribuídas desde a adolescência (4 - 0,79\%), idade adulta (3 - 0,8\%), até a terceira idade (2-3,9\%).

O objetivo central das políticas voltadas para as crianças e adolescentes é de prevenir e eliminar o trabalho desse grupo populacional que, em situação de exploração, compõe a força de 
trabalho, principalmente, no meio rural, distanciando-o do sistema educacional ${ }^{(10)}$. A redução do trabalho infantil no Brasil associa-se à efetivação e universalização destas políticas que provêem algum tipo de renda mínima para as famílias, a exemplo da previdência rural e do programa Bolsa-Familia.

O estudo mostra a incidência de acidentes com idosos trabalhadores rurais, 52 (10,2\%). Nesse sentido, por ser a agricultura familiar brasileira responsável por 38\% do valor bruto da produção de alimentos no país, representando algo em torno de $10 \%$ do Produto Interno Bruto (PIB) agricola, correspondendo a $85 \%$ dos estabelecimentos agropecuários e respondendo por quase $77 \%$ dos postos de trabalho na agricultura, exige uma densa mão de obra, incluindo-se aí a dos mais experientes, a exemplo dos idosos. $\mathrm{Na}$ área rural do país, a contribuição dos idosos no orçamento familiar chegou a $67,3 \%$, dos domicilios em $2007^{(11,12)}$. E, quando os idosos rurais não conseguem mais se manter na labuta diária rural e sem aposentadoria, muitos de seus familiares ficam em situação de extrema pobreza ${ }^{(13)}$.

Observa-se, neste estudo, uma variabilidade no tocante à raça/ coloração da pele: pardos 472 (92,7\%), brancos 21 (4,1\%), negros 10 (2\%) e indigenas $3(0,6 \%)$. Os pardos e negros predominam entre ambos os sexos e em todas as faixas etárias. No que tange à composição racial da população do estado do Ceará, no censo do Instituto Brasileiro de Geografia e Estatística (IBGE) de 2010, 61,8\% de seus habitantes se declararam de cor parda; 32,0\% de cor branca; 4,6\% de cor preta; $1,2 \%$ de cor amarela e $0,23 \%$ se declararam indigenas ${ }^{(14)}$.

Em relação à escolaridade dos trabalhadores rurais, predomina em ambos os sexos, os sujeitos com Ensino Fundamental I incompleto, 32,2\% (164). Apenas 0,6\% (três) cursam ou cursaram o ensino universitário. O analfabetismo ainda é uma realidade nesse meio, 6,9\% (35). O analfabetismo é mais prevalente entre os homens, assim como a pouca escolaridade, o Ensino Fundamental I, principalmente, a partir de 20 anos de idade.

De acordo com o IBGE ${ }^{(15)}$, a frequência escolar de jovens de 15 a 17 anos de idade entre pretos e pardos é de $47,8 \%$, enquanto que a de brancos chega a 62,9\%. Já entre jovens de 18 a 24 anos, os estudantes brancos $(66,6 \%)$ frequentavam o ensino universitário, já os negros ou pardos, apenas 37,4\%, corroborando com o que mostra esse estudo, 40,67\% (207) de adolescentes/jovens e adultos jovens, em sua maioria, pretos e pardos. Observa-se a manutenção das desigualdades que têm, historicamente, limitado o acesso, a progressão e as oportunidades da população negra, de nordestinos e da população rural na educação.

Na Tabela 2, está descrita a situação dos trabalhadores rurais no mercado de trabalho.

Tabela 2 - Descrição da situação dos trabalhadores rurais, com acidente de trabalho, no mercado de trabalho. CEREST Regional de Sobral Ceará, Brasil; número total com estratificação por gênero e idade.

\begin{tabular}{|c|c|c|c|c|c|c|c|c|c|c|c|c|}
\hline \multirow{2}{*}{\multicolumn{3}{|c|}{ Categorias }} & \multicolumn{4}{|c|}{ Gênero } & \multicolumn{6}{|c|}{ Faixa Estária (anos) } \\
\hline & & & \multicolumn{2}{|c|}{ ? } & \multicolumn{2}{|r|}{$?$} & \multicolumn{2}{|c|}{10 a 19} & \multicolumn{2}{|c|}{20 a 59} & \multicolumn{2}{|c|}{60 e mais } \\
\hline Situação no Mercado de Trabalho & $\mathbf{N}$ & $\%$ & $\mathbf{N}$ & $\%$ & $\mathbf{N}$ & $\%$ & $\mathbf{N}$ & $\%$ & $\mathrm{~N}$ & $\%$ & $\mathbf{N}$ & $\%$ \\
\hline Autônomo & 197 & 38,7 & 192 & 39,4 & 5 & 55,6 & 11 & 21,6 & 153 & 37,8 & 33 & 63,5 \\
\hline Empregado não Registrado & 151 & 29,7 & 150 & 30,0 & 1 & 11,1 & 24 & 47,0 & 123 & 30,4 & 4 & 7,7 \\
\hline Trabalho Avulso & 95 & 18,6 & 95 & 19,0 & 0 & 0 & 9 & 17,6 & 78 & 19,3 & 7 & 13,5 \\
\hline Empregado Registrado & 13 & 2,6 & 13 & 2,6 & 0 & 0 & 0 & 0 & 13 & 3,2 & 0 & 0 \\
\hline Aposentado & 4 & 0,8 & 4 & 0,8 & 0 & 0 & 1 & 2,0 & 1 & 0.2 & 0 & 0 \\
\hline Desempregado & 3 & 0,6 & 2 & 0,4 & 1 & 11,1 & 1 & 2,0 & 2 & 0,5 & 0 & 0 \\
\hline Trabalho Temporário & 3 & 0,6 & 3 & 0,6 & 0 & 0 & 0 & 0 & 3 & 0,8 & 0 & 0 \\
\hline Serv. Público Estatutário & 1 & 0,2 & 1 & 0,2 & 0 & 0 & 0 & 0 & 1 & 0,2 & 2 & 3,8 \\
\hline Cooperativado & 1 & 0.2 & 1 & 0,2 & 0 & 0 & 0 & 0 & 1 & 0.2 & 0 & 0 \\
\hline Empregador & 1 & 0,2 & 1 & 0,2 & 0 & 0 & 0 & 0 & 1 & 0,2 & 0 & 0 \\
\hline Outros & 5 & 1,0 & 4 & 0,8 & 1 & 11,1 & 1 & 2,0 & 4 & 1,0 & 0 & 0 \\
\hline Ignorado/branco & 35 & 6,8 & 34 & 6,8 & 1 & 11,1 & 4 & 7,8 & 25 & 6,2 & 6 & 11,5 \\
\hline Total & 509 & 100,0 & 500 & 100,0 & 9 & 100,0 & 51 & 100,0 & 405 & 100,0 & 52 & 100,0 \\
\hline
\end{tabular}

Fonte: Sobral. Secretaria da Saúde. Centro de Referência em Saúde do Trabalhador (CEREST). Sistema Nacional de Agravos de Notificação (SINAN). $2014 .{ }^{\text {? }}$

Observa-se na Tabela 2 que 38,7\% (197) são trabalhadores autônomos, ou seja, aquele profissional que exerce sua atividade sem vínculo empregatício e, em sua grande maioria é do sexo masculino, com 38,4\% (192), na faixa etária de 20 a 59 anos, 37,7\% (153). Outro dado significativo foi o empregado não registrado com 29,7\% (151), com predominância da faixa etária de 20 a 59 anos 30,3\% (123). Em virtude desses trabalhadores não serem registrados, estes têm enormes prejuízos, pois não thes são conferidos direitos importantes como o direito a obter aposentadoria, auxilio-doença e outros benefícios previdenciários, permanecendo em total insegurança caso sofram algum acidente de trabalho. $E$, quando de sua dispensa, não possuem direito a receber as parcelas do seguro-desemprego.
A relação de trabalho precarizado no estudo se mostra em 48,9\% (249) dos sujeitos, ao somatório da associação de empregados sem registro, trabalho avulso e temporário. O trabalho rural, seja ele no campo ou na floresta, apresenta uma situação precária, tanto nas relações como na contratação dos trabalhadores. A estrutura trabalhista está inserida num cenário de intensa desigualdade social, com a desregulamentação desta e a dificuldade de medidas de proteção, a exemplo da fiscalização do trabalho(16).

Tal panorama do mercado de trabalho rural vem sofrendo influências do modelo de produção agrária hegemônico, atualmente instalado no Brasil, sendo este "marcado pela entrada do capitalismo no campo e pela chamada 'Revolução Verde' que lhe dá sustentação, 
tendo um caráter perverso em relação ao modo de apropriação/ exploração/expropriação da natureza e da força de trabalho"(17).

$\mathrm{Na}$ composição da população do estudo, apenas 2,6\% (13) são trabalhadores registrados e $0,8 \%$ (4) aposentados, os trabalhadores registrados estão na faixa etária entre 20 e 59 anos, todos do sexo masculino, reforçando a situação de vulnerabilidade, precarização e falta de valorização do trabalho feminino rural. Quanto aos idosos, $5,2 \%$ (26), apenas quatro são aposentados, representando $0,8 \%$ (quatro) da população do estudo.

O trabalhador rural antes da Constituição Federal de 1988 recebia um benefício assistencial equivalente a cerca de $50 \%$ do salário mínimo vigente e com regras de acesso muito restritas; somente o chefe da família (homem ou mulher), a partir dos 65 anos, desde que comprovado um periodo mínimo de três anos de trabalho no campo tinha o direito de concessão ao benefício rural. Pós 1988, os trabalhadores rurais (homens e mulheres) na categoria de segurados especiais, passaram a ter os mesmos direitos dos trabalhadores urbanos, com o valor do benefício percebido em dobro e as mulheres passaram a ter direito à aposentadoria. A previdência rural leva renda para o campo, garantindo a subsistência de familias que, de outra forma, só obteriam renda durante as colheitas e não o ano todo. Os benefícios passaram de quatro milhões em 1998 para 8,6 milhões em 2011 , cerca de $\mathrm{R} \$ 4,1$ bilhões. Com isso, incluir os trabalhadores rurais na Previdência Social foi um passo importantíssimo em termos de inclusão social((9)

\section{CONCLUSÃO}

O trabalho rural, por sua diversidade produtiva que vai desde o modo de produção artesanal até a mecanização das práticas agropecuárias sem a devida instrução e proteção, tem exposto os trabalhadores e trabalhadoras do campo a situações de vulnerabilidade em suas jornadas laborais, pelas condições insalubres de trabalho e não uso dos EPI. Estas, muitas vezes, são intensificadas pelas práticas abusivas e exploratórias dos grandes latifundiários, que visam apenas o lucro, sem sequer arcar com as despesas previdenciárias para cobertura desses trabalhadores.

Apesar da predominância de homens no trabalho rural, este envolve todo o núcleo familiar, sem distinção de gênero, raça, cor ou idade. As crianças e adolescentes, muitas vezes, abandonam os estudos para ajudar no sustento de suas familias ou são obrigados a assim fazerem, corroborando com o trabalho infantil. As mulheres desempenham papel fundamental na produção agricola, embora não sejam reconhecidas pelo contexto histórico de subordinação, fragilidade. Os idosos ainda representam a força de trabalho rural, mesmo na fase em que deveriam dedicar-se a atividades menos exaustivas e que favoreçam sua qualidade de vida.

Há necessidade de medidas de proteção aos trabalhadores e trabalhadoras rurais, como a fiscalização do trabalho rural e o desenvolvimento de ações voltadas para este público de modo intersetorial a exemplo dos setores Saúde, Assistência Social e agências de extensão rural, dentre outros.

\section{AGRADECIMENTOS}

Fundação Cearense de Apoio ao Desenvolvimento Científico e Tecnológico (FUNCAP).

\section{REFERÊNCIAS}

1. Oliveira JTA. Breve história do trabalho rural no Brasil. [Citado 2015 Jan 21]. Disponivel em: http://www.feagri.unicamp.br/unimac/pdfs/Breve_Historia_do_ Trabalho_Rural_no_Brasil.pdf.

2. Alessi NP, Navarro VL. Saúde e trabalho rural: o caso dos trabalhadores da cultura canavieira na regiäo de Ribeirão Preto, São Paulo, Brasil.Cad. Saúde Pública [online]. 1997 [Citado 2015 Jan 20]; 13(supl. 2): 111-21. Disponivel em: http:// www.scielo.br/pdf/csp/v13s2/1368.pdf.

3. Marques SMT, Silva GP. Trabalho e acidentes no meio rural do Oeste Catarinense - Santa Catarina, Brasil. Rev Bras Saude Ocup. 2003 [Citado 2015 Jan 21]; 28(1078): 101-05. Disponivel em: http://www.scielo.br/pdf/ rbso/v28n107-108/09.pdf

4.Scheil-Adlung, X. (Ed.). Global evidence on inequities in rural health protection: new data on rural deficits in health coverage for 174 countries; International Labour Office, Social Protection Department. - Geneva: ILO; 2015. (Extension of Social Security series; No. 47).

5. Brasil. Ministério da Saúde. Portaria NNo 1823/GM, de 23 de agosto de 2012 - Institui a Política Nacional de Saúde do Trabalhador e da Trabalhadora. Diário Oficial [da] República Federativa do Brasil, n`215-E, Poder Executivo, Brasilia, DF, 24 de Agosto de 2012. Seção 1, p. 46.

6. Brasil. Ministério da Saúde. Política Nacional de Saúde Integral das Populações do Campo, da Floresta e das Águas. Brasilia-DF: Ministério da Saúde, 2013.

7. Sobral. Secretaria da Saúde. Centro de Referência em Saúde do Trabalhador Sistema Nacional de Agravos de Notificação. Acidentes de Trabalho Grave com trabalhadores rurais. Sobral: Secretaria da Saúde, 2014.

8. Brasil. Ministério da Saúde. Secretaria de Vigilância em Saúde. Guia de Vigilância em Saúde. Brasilia: Ministério da Saúde; 2014

9.Abramo L. Introdução. In: Organização Internacional do Trabalho. Igualdade de gênero e raça no trabalho: avanços e desafios. Brasilia: OIT; 2010. p. 15-47.
10. Conde SF. As medidas de enfrentamento à exploração do trabalho infantil no Brasil: forças em luta. Rev Katal, 2013; 16(2): 241-7.

11. Silva CBC, Schneider S. Gênero, trabalho rural e pluriatividade. In: Scott P. Cordeiro R, Menezes M (Org.) Gênero e Geração em Contextos Rurais. Florianópolis - SC: Ed. Mulheres, 2010, p. 183-207.

12. Instituto de Pesquisa e Estratégia Econômica do Ceará (IPECE). Perfil da Raça da População Cearense: Análise a partir dos dados do Censo Demográfico 2010. Informe № 23. 2012. [Citado 2015 Nov 09]. Disponivel em: http://www. ipece.ce.gov.br/publicacoes/ipeceinforme/Ipece_Informe_23 fevereiro_2012.pdf

13. Tavares VO, Teixeira KMD, Wajnman S, Loreto MDS. Interfaces entre a renda dos idosos aposentados rurais e o contexto familiar. Textos \& Contextos. 2011. 10(1): $94-108$.

14. Instituto de Pesquisa e Estratégia Econômica do Ceará. Perfil da Raça da População Cearense: Análise a partir dos dados do Censo Demográfico 2010. Informe № 23. 2012. [Citado 2015 Nov 09]. Disponivel em: http://www.ipece. ce.gov.br/publicacoes/ipeceinforme/Ipece_Informe_23_fevereiro_2012.pdf

15. Brasil. Ministério do Planejamento, Orçamento e Gestão. IBGE. Sintese de indicadores sociais: uma análise das condições de vida da população brasileira 2013. Rio de Janeiro: IBGE; 2013

16. Araújo ACP, Nogueira DP, Augusto LGS. Impacto dos praguicidas na saúde: estudo da cultura de tomate. Rev Saude Publica [online]. 2000 [Citado 2015 Jan 10]: 34 (3): 309-13. Disponivel: http://www.scielo.br/pdf/rsp/v34n3/2235.pdf.

17. Augusto LGS, Carneiro FF, Pignati W, Rigotto RM, Friedric HK, Faria NMX et al. Dossiê ABRASCO - Um alerta sobre os impactos dos agrotóxicos na saúde. Parte 2 - Agrotóxicos, Saúde, Ambiente e Sustentabilidade. Rio de Janeiro: ABRASCO 2012. 